\title{
Biomimetics of Bone Implants: The Regenerative Road
}

\author{
Elizabeth Brett, John Flacco, Charles Blackshear, Michael T. Longaker,,2 and Derrick C. Wan ${ }^{1, *}$
}

\begin{abstract}
The current strategies for healing bone defects are numerous and varied. At the core of each bone healing therapy is a biomimetic mechanism, which works to enhance bone growth. These range from porous scaffolds, bone mineral usage, collagen, and glycosaminoglycan substitutes to transplanted cell populations. Bone defects face a range of difficulty in their healing, given the composite of dense outer compact bone and blood-rich inner trabecular bone. As such, the tissue possesses a number of inherent characteristics, which may be clinically harnessed as promoters of bone healing. These include mechanical characteristics, mineral composition, native collagen content, and cellular fraction of bone. This review charts multiple biomimetic strategies to help heal bony defects in large and small osseous injury sites, with a special focus on cell transplantation.
\end{abstract}

Keywords: biomimetic; bone graft; implant; osteoconduction; osteoinduction; stem cell

\section{Introduction}

Biomimetics and biomimicry are thought processes applied to biomaterial design, where materials meant for implantation have properties, which mirror closely those of natural material. Design of implants in this manner can circumvent some of the roadblocks in synthetic biomaterial design and function. ${ }^{1}$ This review will highlight the variety of biomimetic biomaterial research, with a specific focus on bone regeneration.

Clinically, a biomaterial needs to predictably accomplish its role $100 \%$ of the time. In recent years, biomimetic R\&D has identified issues with existing materials and focused on aspects of implants such as physical shape, surface chemistry, and mechanical properties. Deleterious outcomes of implants, biologic or synthetic (peri-implantitis, peri-mucositis, peri-implant disease, infection), can mean pain, mechanical loosening, failure, and eventual need for extraction. ${ }^{2,3}$ Similarly, some literature has outlined concerns with implants, specifically showing unfavorable physical remodeling over time. ${ }^{4}$ Eliminating variability of results in biomaterial implantation means a huge decrease in patient morbidity and associated costs. These concerns have ushered biomimetics and new material design techniques to the forefront.

Bone is one of the few tissues capable of complete regeneration in adult humans. As such, bone healing has several distinct aspects of healing, which translate very well to biomimetic biomaterial design. Osteoconductive scaffolds, osteoinductive stimuli, and potent osteogenic cell populations are options for a therapy, in which cells, extracellular matrices (ECM), and chemical signaling act in concert to rapidly heal bone. ${ }^{5}$

It is important to consider the material breakdown of bone to best replace like with like. Eighty percent of bone is made up of the outer, compact (cortical) bone, while $20 \%$ remaining is inner, spongy (trabecular) bone. ${ }^{6}$ Therefore, analyzing the content of compact bone will focus the therapy on healing the larger portion of the bone. Knowing that compact bone is $70 \%$ inorganic mineral (chiefly hydroxyapatite), 22\% organic protein (collagen, cells, hyaluronic acid [HA]), and $8 \%$ water allows for bone graft design to focus on one compartment ${ }^{7}$ (Fig. 1).

\footnotetext{
${ }^{1}$ Hagey Laboratory for Pediatric Regenerative Medicine, Plastic and Reconstructive Surgery Division, Department of Surgery, Stanford University School of Medicine, Stanford, California.

${ }^{2}$ Institute for Stem Cell Biology and Regenerative Medicine, Stanford University, Stanford, California.

*Address correspondence to: Derrick C. Wan, MD, Hagey Laboratory for Pediatric Regenerative Medicine, Plastic and Reconstructive Surgery Division, Department of Surgery, Stanford University School of Medicine, 257 Campus Drive, Stanford, CA, E-mail: dwan@stanford.edu
}

(c) Elizabeth Brett et al. 2017; Published by Mary Ann Liebert, Inc. This Open Access article is distributed under the terms of the Creative Commons License (http://creativecommons.org/licenses/by/4.0), which permits unrestricted use, distribution, and reproduction in any medium, provided the original work is properly credited. 


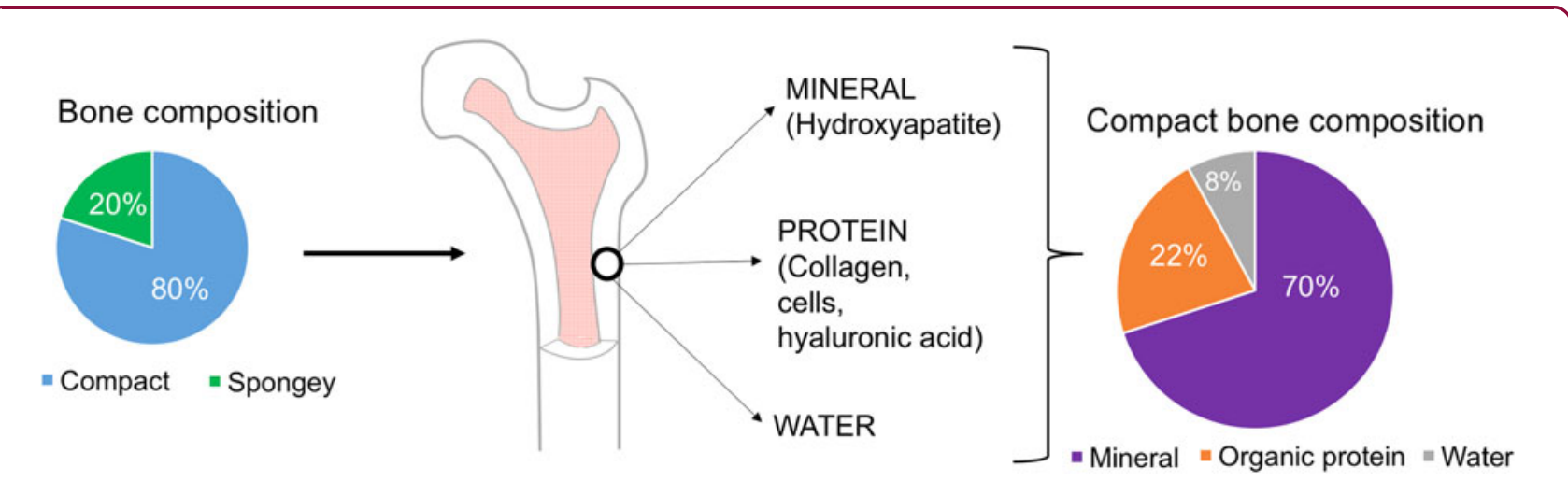

FIG. 1. Schematic showing composition of bone between compact (cortical) and spongy (cancellous). Cortical bone is evaluated further and broken into its constituents; $70 \%$ mineral, $22 \%$ organic protein, $8 \%$ water.

\section{The Recipient Site}

Types of bony reconstruction range from structural nonload-bearing bone (e.g., calvarium) to larger compact load-bearing bone (e.g., femur). Host bone can often present an orthopedic challenge. Osteoporotic bone will be a weak tissue to host implant hardware. ${ }^{8}$ Similarly, osteomyelitic bone is a major contraindication for implants, considering the likelihood of infectious staphylococci biofilm formation. ${ }^{9,10}$ To ensure a fit of the implant in the host bone, under-reaming is often performed. This is a drilling process designed to facilitate the implant, which is sometimes known to fracture the host bone further. ${ }^{11}$ It is especially problematic in the context of osteoporotic bone, which can exhibit low "pull out strength" of an implant once in situ due to insufficient cortical bone. ${ }^{12}$ Quite common also is the issue of poor nutrient diffusion, borne of compromised blood supply. Diffusion distance of oxygen in vivo is $150-200 \mu \mathrm{m} .^{13}$ As such, certain materials operate via stimulation of vascular ingrowth into the host bone. Use of cobalt ions as inactivators of prolyl hydroxylase has been shown to stabilize HIF-1 $\alpha$, a potent proangiogenic factor, resulting in upregulated expression of genes such as GLUT1, erythropoietin, VEGF, and $P D G F .{ }^{14,15}$ Bone that has undergone irradiation is an example of a hostile recipient site. There is conflicting retrospective literature, which debates the use of hyperbaric oxygen (HBO) in treating host bone, specifically during craniofacial reconstruction after head and neck cancer. Some studies show improved vascularity and increased basic fibroblast growth factor of grafted recipient bone, ${ }^{16}$ and other studies exhibit no significant difference in graft take with HBO. ${ }^{17}$ Macroscopically, there is reliance on mechanical design of threaded implants to give the implant mechanical integration. The ability to screw an im- plant through bone gives immediate stability and provides close, fixed contact between the graft and host bone.

\section{Mechanical Stability of Implant}

Issues with bone implants chiefly lie in materials destined for load-bearing bone healing. The Young's modulus of trabecular bone is $10.4-14.8 \mathrm{GPa}$, and cortical bone measures $18.4-20.7 \mathrm{GPa}^{18}$ These values differ drastically from those of commonly used metals for bone implants; stainless steel measuring $180 \mathrm{GPa}$, cobalt chromium $(\mathrm{Co}-\mathrm{Cr})$ at $210 \mathrm{GPa}$, and titanium at $110 \mathrm{GPa} .{ }^{19}$ Stress shielding is the physiological response resulting from implanting a harder material into a softer host tissue. The physiological result manifests in fibrous encapsulation of implant where possible, allowing for micromovement of the fibrotic sheath around the implant. Mobility of an implant in situ on the order $<100 \mu \mathrm{m}$ creates a specific wear, called fretting. ${ }^{20}$ Fretting implants can gradually loosen and eventually fail within the host bone. ${ }^{21}$ Moreover, fragments of the implant are frequently known to break off the body of the implant, causing local abrasion of the surrounding, softer bone tissue. Macrophages have been seen to internalize particles of polyethyleneimine surrounding total hip arthroplasties. ${ }^{22}$ Furthermore, debris of implants can sometimes be found in alternate locations in the body, such as the spleen, liver, and abdominal lymph nodes of arthroplasty patients. ${ }^{23}$

It was Branemark in 1981 who first showed the complete "osseointegration" of titanium. During a fracture fixation experiment in a rabbit femur, Branemark discovered the removal of the titanium implant from bone was impossible. Further studies using transmission electron microscopy showed direct contact between bone and implant, without the surrounding fibrous capsule 
responsible for implant looseness and micromovement. ${ }^{24}$ Filamentous collagen type 1 was found to form fibrils at the implant-bone interface resembling the strong Sharpey's fibers of the scalp, explaining the tight coupling of metal and bone. ${ }^{25}$ The phenomenon of the absence of fibrous encapsulation around titanium implants has proven significantly important in implant design in more than just bone. Tissue expanders used for breast reconstruction can include a titanium-coated mesh to reduce the fibrotic content of the breast. ${ }^{26}$ In the context of bone, titanium is now being tested in advanced models of craniofacial bone healing, using porous titanium granules as new-bone regeneration stimuli to recreate maxillary sinuses. ${ }^{27}$ However, in terms of biomimetics, titanium presents some mechanical challenges, specifically in terms of difference in stiffness between it and the host tissue, for example, femur. This hurdle is called modular mismatch ${ }^{28}$ and can be circumvented by utilizing material whose bulk mechanical properties more closely resemble bone.

\section{Grafts Based on Bone Mineral Components}

The unique nature of bone is that it is largely mineralized. In its dry mass, bone is $60-70 \%$ mineral, which is nonimmunogenic and ubiquitously found. ${ }^{29}$ As such, the use of natural material already in existence presents an option for creating biomimetic implant matter. Nacre, or mother-of-pearl, is pure calcium carbonate produced by molluscs. Mixing pulverized nacre with patient blood and implanting the mixture into a human mandibular defect site was largely effective in closing the defect and stimulating regenerative cellular activity in the location. ${ }^{30}$ Many successful bone biomaterials incorporate calcium or hydroxyapatite to help facilitate bone formation and graft "take." A study comparing biologic bovine-derived bone grafts (BioOss ${ }^{\circledR}$ Bone Substitute; Ed. Geistlich Soehne, Wolhusen, Switzerland), with a highly porous synthetic hydroxyapatite scaffold (IngeniOs ${ }^{\mathrm{TM}} \mathrm{HA}$ Synthetic Bone Particles; Zimmer Dental, Inc., Carlsbad, CA), showed highly similar chemistry, morphology, and structure with the exception of crystallinity. ${ }^{27}$ FT-IR spectra revealed high crystallinity (thus low resorption) of the synthetic IngeniOs Hydroxyapatite Synthetic Bone Particles, owing to the purity of its manufacturing processes versus natural variation and other trace elements inherent to biologic grafts. ${ }^{31}$ The difference in purity between synthetic and biologic material by proxy of uniform industrial manufacture is seen throughout most implant forms.
Beta tricalcium phosphate $(\beta-\mathrm{TCP})$ is a calcium salt abundant in bone, and has been shown to be highly and quickly reactive as part of a bone graft. This is because in an aqueous environment, TCP reacts to form hydroxyapatite. ${ }^{32}$ Effects of TCP can be exaggerated with strategic addition of growth factors in vivo, as was seen throughout a series of randomized control trials using platelet-derived growth factor and $\beta$-TCP as agents to heal periodontal intraosseous defects. ${ }^{33}$ In fact, $\beta$-TCP has been shown to contribute to bone healing faster than hydroxyapatite alone, secondary to its rapid rate of resorption. ${ }^{34}$ Meanwhile, HAPEX is an amalgamation of the biologic mineral hydroxyapatite and synthetic high-weight polyethylene. This mixture makes for a bioactive polymer, which has been used in the reconstruction of orbital floor and middle ear defects. ${ }^{35}$

The ideal bone implant will resorb completely after a time of osteoinduction or osteoconduction of surrounding healing tissue. Most bone healing substitutes today incorporate a biologic component, intended to mimic native bone mechanical structure or mineral chemistry. Cerasorb is pure $\beta$-TCP, designed to be mixed with the patient's own blood or platelet-rich plasma and added to the defect site, primarily for periodontic healing (http://curasaninc .com/products/cerasorb). Alternatively, natural bone biomimetics have been found in coral, for both chemistry and structure. Coral forms hydroxyapatite on its surface due to its calcium carbonate core and also retains its native trabecular structure, having inherent biomedical value as a spongy bone substitute. ${ }^{36,37}$

\section{Grafts Based on Structural Protein}

To further the biomimetic approach of bone grafts, materials are being designed with a microscopic eye on ECM. These incorporate more biologic factors than synthetic, such as endogenous protein, collagen, and HA. HA is a high-molecular-weight nonsulfated glycosaminoglycan, which is formed in the plasma membrane of cells. ${ }^{38}$ Many biomimetic ECM scaffolds containing HA have reached the market for dermal applications, showing hyaluronate as an antifibrotic hydrating agent in a healing wound. ${ }^{39}$ However, in the context of bone grafts, rabbit tibias, which received HA, showed increased healing 20 days after injury, showing fibrocartilage formation, which later ossified, in comparison to the non-HA-treated bones, which formed purely fibrous unions. ${ }^{40}$ More sophisticated, combinatorial approaches have been performed, involving collagenase to stimulate mandibular bone remodeling, and HA in a hydrogel with calcium sulfide hemihydrate; a bioresorbable, osteoconductive compound. ${ }^{41}$ 
Composite biomimetic grafts over simple hydrogel injection are required for healing of large load-bearing bones. For instance, addition of protein to a hydrogel or impregnated into an implant introduces cellular osteogenic mechanisms. In a canine femoral defect model, bone morphogenetic protein (BMP) in tandem with collagen type-1/TCP showed increased healing of the femur in the presence of bone marrow aspirate. ${ }^{42}$ This study points to the use of collagen type 1 to match the modulus of the femur, and the utility of bone marrow-derived cells stimulated by pro-osteogenic growth factors in bone healing.

A highly osteogenic component of bone is the periosteum, a stratified structure of an inner cell layer (cambium layer), and tough, fibrous outer layer. ${ }^{43}$ In fact, damaged bone, which undergoes delayed reconstruction, often exhibits heterotopic ossification; new disorganized bone formation at the injury site as a result of damaged periosteum. ${ }^{44}$ As such, strategically placed periosteal grafts present a highly biomimetic solution of autografting onto damaged bone. ${ }^{45}$ However, due to the sheet-like structure of the periosteum, periosteal grafts have found their utility primarily in dental and alveolar healing, as opposed to long, load-bearing bone reconstruction. ${ }^{46}$

\section{Grafts Based on Cellular Implants}

Despite orthopedic management of fractures becoming better and better, some healed injuries will persist with fibrous nonunions, ${ }^{47}$ an issue that may still be encountered clinically. To address this, "The Diamond Concept" has been reported, which encompasses four different aspects of in vivo bone regeneration; an osteoconductive scaffold, a suitable mechanical environment, osteoinductive signals, and a pro-osteogenic cell population. ${ }^{5}$

The ability to direct stem cells to an osteogenic pathway represents a huge regenerative medical role. Addition of cells to bone grafts is a concept based on isolating cell types, which will either immediately and directly add bone and aid in the remodeling procedure (osteoblasts/osteoclasts), or have the potential to differentiate, affording the healing tissue with angiogenic and osteogenic factors (mesenchymal stem cells [MSCs] and adipose-derived stem cells $[\mathrm{ASCs}]) .{ }^{48}$ Moreover, there are now established surface protein expression profiles to identify heterogeneous stem cells, which are more likely to differentiate into bone.

Cell surface markers indicative of osteogenic behavior can vary from poorly defined CD markers to more wellknown pro-osteogenic growth factor receptors. BMP receptor type-1b (BMPR-1b) binds BMP and has an important role in directing bone formation. ${ }^{49}$ As such, using FACS to select for BMPR-1b-positive cells from ASCs results in a population with increased osteogenic gene expression and in vitro osteogenic potential. ${ }^{50}$ Moreover, when coupled with a porous, osteoconductive scaffold coated in osteoinductive hydroxyapatite, rapid bone formation is observed in vivo. ${ }^{51}$ Similarly, FACS sorted ASCs positive for CD90 (Thy-1) have been shown to significantly increase healing of bone defects when compared with their negative and unsorted control groups. ${ }^{52,53}$ Importantly, CD90 expression has been shown to vary dramatically between in vivo and in vitro settings, making use of this marker unpredictable. ${ }^{54}$ Similarly, other markers also have difficult expression profiles to track and analyze, such as CD105 (endoglin), a bone marrow mesenchymal cell marker. Expression of CD105 in freshly harvested ASCs is extremely low, proceeded by near ubiquitous expression after 4-7 days in culture. However, it was discovered that isolating CD105-negative cells after $36 \mathrm{~h}$ in culture yielded a subpopulation with enhanced osteogenic potential in vivo. ${ }^{55} \mathrm{CD} 105$ is especially nuanced, although in that it acts as a coreceptor for TGF- $\beta 1$, which is a known antagonist of osteogenic differentiation, ${ }^{56}$ thus, explaining a parallel decrease in bone formation with increased expression. These findings simultaneously highlight the promise and problems of using surface marker selection criteria in isolating heterogeneous stem cell populations for bone regenerative purposes.

There are multiple different bone diseases being researched under an autologous cell transfer lens. For instance, the efficacy of bone regeneration by autologous bone marrow harvested from the anterior iliac crest has been shown in atrophic diaphyseal nonunion. Here, a biomaterial was created by concentrating marrow via centrifugation, which could be loaded into a syringe and injected into the recipient site. Analysis of diseased tibias postmarrow transplant showed increased bone callus mineralization. ${ }^{57}$ Similarly, osteogenesis imperfecta (OI) is a genetic disease of the mesenchymal cells, whereby a defective collagen type 1 is produced, giving rise to bone weakness and malformation. Unmanipulated bone marrow donations from healthy matched siblings or family members have been shown to increase trabecular bone formation in the recipient OI patient. ${ }^{58}$ On the cellular level, the harvest, culture, and transplant of bone marrow-derived stromal cells have been found to be effective in repairing large bone defects in humans. ${ }^{59}$ However, these cells 
are most efficient when placed in situ seeded on a macroporous scaffold. ${ }^{60}$

Combinations of cell populations with scaffolds have shown efficacy in healing bone defects. It was seen in composite grafts of BMP-2, HA-based hydrogel, and hMSCs that there was a synergistic effect of the group, which received cells over those that did not, in the healing of rat calvarial defects. ${ }^{61}$ In a human pediatric case study, autologous ASCs used with autologous fibrin glue (derived from patient's own blood) were used successfully together to heal the patient's widespread calvarial defects. ${ }^{62}$ From an endochondral ossification perspective, cell culture offers a biomimetic tissue engineering route. Culturing chondrocytes on a porous polylactic acid scaffold revealed deposition of collagen type II and glycosaminoglycans, mimicking the in vivo histology of cartilage. ${ }^{63}$ The use of specialized cell populations grown on scaffolds can be a future strategic method of stimulating bone/cartilage formation in vivo.

\section{Conclusion}

To meet key requirements of bone regeneration, we need to move beyond the current standards of therapies and toward regenerative strategies. Biomimetics allows us to learn from and emulate the inherently self-sufficient healing mechanisms already in place. Due to the biological nature of much of the research mentioned in this review, product development still faces considerable regulatory hurdles. However, the knowledge bank, which is borne of this research, has allowed extremely effective biologic treatments to be developed. The biomimetic boundaries that can be pushed belong to implanted biological matter; collagen or collagen analogs, soluble minerals, or active cell populations.

\section{Acknowledgments}

M.T.L. was supported by NIH grants, U01 HL099776, R01 DE021683, the Oak Foundation, Hagey Laboratory for Pediatric Regenerative Medicine, and the Gunn/Olivier Fund. D.C.W. was supported by NIH grant 1K08 DE024269-01, the Hagey Laboratory for Pediatric Regenerative Medicine, and the Stanford University Child Health Research Institute Faculty Scholar Award.

\section{Author Disclosure Statement}

No competing financial interests exist.

\section{References}

1. Kumar $P$, Vinitha B, Fathima G. Bone grafts in dentistry. J Pharm Bioallied Sci. 2013;5(Suppl 1):S125-S127.

2. Esposito $M$, et al. Biological factors contributing to failures of osseointegrated oral implants. (I). Success criteria and epidemiology. Eur J Oral Sci. 1998; 106:527-551.

3. Cevasco M, Itani KM. Ventral hernia repair with synthetic, composite, and biologic mesh: characteristics, indications, and infection profile. Surg Infect (Larchmt). 2012;13:209-215.

4. Cavallaro A, et al. Use of biological meshes for abdominal wall reconstruction in highly contaminated fields. World J Gastroenterol. 2010;16:1928-1933.

5. Giannoudis PV, Einhorn TA, Marsh D. Fracture healing: the diamond concept. Injury. 2007;38(Suppl 4): S3-S6.

6. Declercq HA, et al. The role of scaffold architecture and composition on the bone formation by adipose-derived stem cells. Tissue Eng Part A. 2014;20:434-444.

7. Arthur C, Hall JEG. Textbook of Medical Physiology. Elsevier, Saunders, Philadelphia, PA, 2005.

8. Merheb J, et al. Influence of skeletal and local bone density on dental implant stability in patients with osteoporosis. Clin Implant Dent Relat Res. 2016;18:253-260.

9. Funao $\mathrm{H}$, et al. A novel hydroxyapatite film coated with ionic silver via inositol hexaphosphate chelation prevents implant-associated infection. Sci Rep. 2016:6:23238.

10. von Eiff C, Peters G, Heilmann C. Pathogenesis of infections due to coagulase-negative staphylococci. Lancet Infect Dis. 2002;2:677-685.

11. Winter W, Karl M. Basic considerations for determining the amount of press fit in acetabular cup endoprostheses as a function of the elastic bone behavior. Biomed Tech (Berl). 2014;59:413-420.

12. Pesce V, et al. Surgical approach to bone healing in osteoporosis. Clin Cases Miner Bone Metab. 2009;6:131-135.

13. Laschke MW, et al. Angiogenesis in tissue engineering: breathing life into constructed tissue substitutes. Tissue Eng. 2006;12:2093-2104.

14. Wu C, et al. Hypoxia-mimicking mesoporous bioactive glass scaffolds with controllable cobalt ion release for bone tissue engineering. Biomaterials. 2012;33:2076-2085.

15. Wu Y, et al. Mesenchymal stem cells enhance wound healing through differentiation and angiogenesis. Stem Cells. 2007;25:2648-2659.

16. Zheng $M$, et al. How to improve the survival rate of implants after radiotherapy for head and neck cancer? J Periodontal Implant Sci. 2014:44:2-7.

17. Chambrone $L$, et al. Dental implants installed in irradiated jaws: a systematic review. J Dent Res. 2013;92(Suppl 12):119S-130S.

18. Rho JY, Ashman RB, Turner CH. Young's modulus of trabecular and cortical bone material: ultrasonic and microtensile measurements. J Biomech. 1993;26:111-119.

19. Niinomi $M$, Nakai $M$. Titanium-based biomaterials for preventing stress shielding between implant devices and bone. Int J Biomater. 2011;2011:836587.

20. Gao SS, et al. Micromotions and combined damages at the dental implant/bone interface. Int J Oral Sci. 2012;4:182-188.

21. Muller $\mathrm{M}$, et al. Bone-implant interface shear modulus and ultimate stress in a transcortical rabbit model of open-pore Ti6Al4V implants. J Biomech. 2006;39:2123-2132.

22. Schmalzried TP, Jasty M, Harris WH. Periprosthetic bone loss in total hip arthroplasty. Polyethylene wear debris and the concept of the effective joint space. J Bone Joint Surg Am. 1992;74:849-863.

23. Urban RM, et al. Dissemination of wear particles to the liver, spleen, and abdominal lymph nodes of patients with hip or knee replacement. J Bone Joint Surg Am. 2000;82:457-476.

24. Duyck J, et al. The influence of micro-motion on the tissue differentiation around immediately loaded cylindrical turned titanium implants. Arch Oral Biol. 2006;51:1-9.

25. Albrektsson T, et al. Osseointegrated titanium implants. Requirements for ensuring a long-lasting, direct bone-to-implant anchorage in man. Acta Orthop Scand. 1981;52:155-170.

26. Casella $D$, et al. Subcutaneous tissue expander placement with synthetic titanium-coated mesh in breast reconstruction: long-term results. Plast Reconstr Surg Glob Open. 2015;3:e577. 
27. Dursun CK, et al. Effect of porous titanium granules on bone regeneration and primary stability in maxillary sinus: a human clinical, histomorphometric, and microcomputed tomography analyses. J Craniofac Surg. 2016;27:391-397.

28. Gruen TA, McNeice GM, Amstutz HC. "Modes of failure" of cemented stem-type femoral components: a radiographic analysis of loosening. Clin Orthop Relat Res. 1979;141:17-27.

29. Boskey AL. Bone composition: relationship to bone fragility and antiosteoporotic drug effects. Bonekey Rep. 2013;2:447

30. Westbroek P, Marin F. A marriage of bone and nacre. Nature. 1998;392:861-862.

31. Kattimani VS, et al. Comparative evaluation of bovine derived hydroxyapatite and synthetic hydroxyapatite graft in bone regeneration of human maxillary cystic defects: a clinico-radiological study. Indian J Dent Res. 2014:25:594-601.

32. Cao H, Kuboyama N. A biodegradable porous composite scaffold of PGA $\beta$-TCP for bone tissue engineering. Bone. 2010;46:386-395.

33. Calin C, Patrascu I. Growth factors and beta-tricalcium phosphate in the treatment of periodontal intraosseous defects: a systematic review and meta-analysis of randomised controlled trials. Arch Oral Biol. 2016;66: 44-54.

34. Walsh WR, et al. Beta-TCP bone graft substitutes in a bilateral rabbit tibia defect model. Biomaterials. 2008;29:266-271.

35. Tanner KE. Bioactive ceramic-reinforced composites for bone augmentation. J R Soc Interface. 2010;7(Suppl 5):S541-S557.

36. Vago $R$, et al. Hard tissue remodeling using biofabricated coralline biomaterials. J Biochem Biophys Methods. 2002;50:253-259.

37. Damien E, Revell PA. Coralline hydroxyapatite bone graft substitute: a review of experimental studies and biomedical applications. J Appl Biomater Biomech. 2004;2:65-73.

38. Serban MA, Prestwich GD. Modular extracellular matrices: solutions for the puzzle. Methods. 2008;45:93-98.

39. Davidson JM, et al. Hyaluronate derivatives and their application to wound healing: preliminary observations. Clin Mater. 1991;8: $171-177$.

40. Aslan M, Simsek G, Dayi E. The effect of hyaluronic acid-supplemented bone graft in bone healing: experimental study in rabbits. J Biomater Appl. 2006;20:209-220.

41. Subramaniam $S$, et al. Hydroxyapatite-calcium sulfate-hyaluronic acid composite encapsulated with collagenase as bone substitute for alveolar bone regeneration. Biomaterials. 2016;74:99-108.

42. Luangphakdy V, et al. Evaluation of rhBMP-2/collagen/TCP-HA bone graft with and without bone marrow cells in the canine femoral multi defect model. Eur Cell Mater. 2015;29:57-68; discussion 68-69.

43. Finley JM, Acland RD, Wood MB. Revascularized periosteal grafts - a new method to produce functional new bone without bone grafting. Plast Reconstr Surg. 1978;61:1-6.

44. Ueno T, et al. Cellular origin of endochondral ossification from grafted periosteum. Anat Rec. 2001;264:348-357.

45. Zhang $X$, et al. A perspective: engineering periosteum for structural bone graft healing. Clin Orthop Relat Res. 2008;466:1777-1787.

46. Mahajan A. Periosteum: a highly underrated tool in dentistry. Int J Dent. 2012;2012:717816.

47. Gómez-Barrena $E$, et al. Bone fracture healing: cell therapy in delayed unions and nonunions. Bone. 2015;70:93-101.

48. Liao HT, Chen CT. Osteogenic potential: comparison between bone marrow and adipose-derived mesenchymal stem cells. World J Stem Cells. 2014;6:288-295.

49. Wan DC, et al. Osteogenic differentiation of mouse adipose-derived adult stromal cells requires retinoic acid and bone morphogenetic protein receptor type IB signaling. Proc Natl Acad Sci U S A. 2006;103: $12335-12340$.

50. Quarto N, Wan DC, Longaker MT. Molecular mechanisms of FGF-2 inhibitory activity in the osteogenic context of mouse adipose-derived stem cells (mASCs). Bone. 2008;42:1040-1052.

51. McArdle $A$, et al. Positive selection for bone morphogenetic protein receptor type-IB promotes differentiation and specification of human adipose-derived stromal cells toward an osteogenic lineage. Tissue Eng Part A. 2014;20:3031-3040.

52. Chung MT, et al. CD90 (Thy-1)-positive selection enhances osteogenic capacity of human adipose-derived stromal cells. Tissue Eng Part A. 2013;19:989-997.
53. Yamamoto $\mathrm{M}$, et al. Osteogenic potential of mouse adipose-derived stem cells sorted for CD90 and CD105 in vitro. Stem Cells Int 2014;2014:576358.

54. Wiesmann A, et al. Decreased CD90 expression in human mesenchymal stem cells by applying mechanical stimulation. Head Face Med 2006;2:8.

55. Levi B, et al. CD105 protein depletion enhances human adipose-derived stromal cell osteogenesis through reduction of transforming growth factor beta1 (TGF-beta1) signaling. J Biol Chem. 2011;286:39497-39509.

56. Zhang $\mathrm{L}$, et al. Modulation of TGF- $\beta$ signaling by endoglin in murine hemangioblast development and primitive hematopoiesis. Blood. 2011;118:88-97.

57. Hernigou $P$, et al. Percutaneous autologous bone-marrow grafting for nonunions. Influence of the number and concentration of progenitor cells. J Bone Joint Surg Am. 2005;87:1430-1437.

58. Horwitz EM, et al. Transplantability and therapeutic effects of bone marrow-derived mesenchymal cells in children with osteogenesis imperfecta. Nat Med. 1999;5:309-313.

59. Quarto R, et al. Repair of large bone defects with the use of autologous bone marrow stromal cells. N Engl J Med. 2001;344:385-386.

60. Martin I, et al. Fibroblast growth factor-2 supports ex vivo expansion and maintenance of osteogenic precursors from human bone marrow. Endocrinology. 1997;138:4456-4462.

61. Kim J, et al. Bone regeneration using hyaluronic acid-based hydrogel with bone morphogenic protein-2 and human mesenchymal stem cells. Biomaterials. 2007;28:1830-1837.

62. Lendeckel S, et al. Autologous stem cells (adipose) and fibrin glue used to treat widespread traumatic calvarial defects: case report. J Craniomaxillofac Surg. 2004;32:370-373.

63. Langer R, Vacanti JP. Tissue engineering. Science. 1993;260:920-926.

Cite this article as: Brett E, Flacco J, Blackshear C, Longaker MT,

Wan DC (2017) Biomimetics of bone implants: the regenerative road, BioResearch Open Access 6:1, 1-6, DOI: 10.1089/biores.2016.0044.

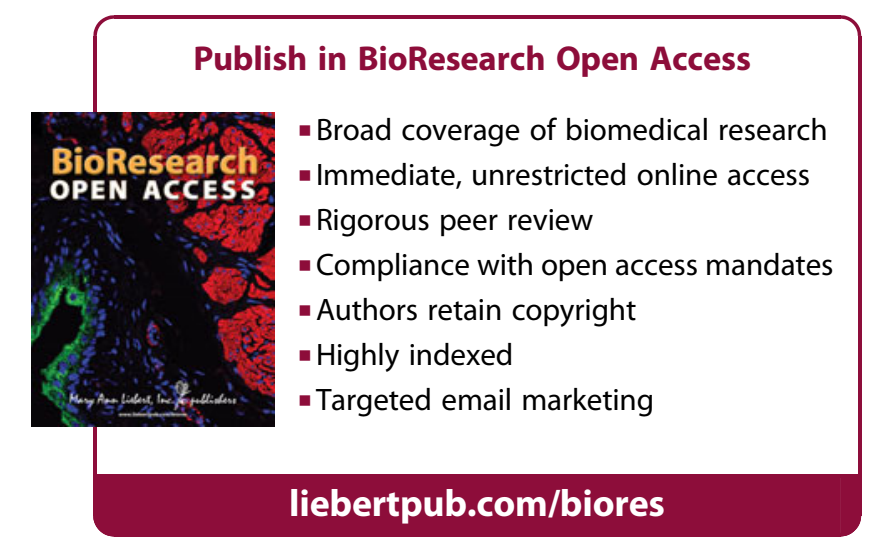

